\title{
Notes \\ An assessment of some closed-form expressions for the Voigt function II: Utilizing rational approximations for the Gauss function
}

\author{
Franz Schreier \\ DLR - German Aerospace Center, Remote Sensing Technology Institute, \\ Oberpfaffenhofen, 82234 Weßling, Germany
}

\begin{abstract}
Rational approximations for the Gauss function can be used to construct closedform expressions of the Voigt function $K(x, y)$ in terms of rational functions, logarithms and inverse trigonometric functions. The comparison with accurate reference values indicates a relative accuracy in the percent range for $y \gtrsim 1$, but serious problems for smaller $y$. Furthermore, these expressions are not competitive with other algorithms with respect to computational speed. Both accuracy and speed tests indicate that supposedly "good" approximations of the integrand do not necessarily provide good approximations of the integral, i.e. Voigt function.
\end{abstract}

Keywords: Complex error function; Complex probability function; Plasma dispersion function; Faddeyeva function

$P A C S$ :

02.30.Mv Approximations and expansions

02.60.-x Numerical approximation and analysis

02.70.-c Computational techniques

\footnotetext{
* Corresponding author

Email address: franz.schreier@dlr.de (Franz Schreier)
} 


\section{Introduction}

The convolution integral of a Lorentzian and Gaussian function is commonly known as the Voigt function [1] and important in many branches of physics and related fields. Because the integral does not have a closed-form analytical solution (i.e. it cannot be expressed in finite terms of elementary functions), computational approaches have been discussed in numerous papers. Most "state-ofthe art" modern algorithms evaluate the closely related complex error function (also known as complex probability function, plasma dispersion function, or Fadde(ye)va function, cf. e.g., 2 4). Several authors have developed approximations to the complex error function or its real part, the Voigt function, using appropriate representations of the Gaussian. Salzer [5] and Zaghloul and Ali 6] expressed the Gaussian as an infinite sum of hyperbolic cosines with exponential weights, Rybicki [7] wrote the Gaussian as an infinite sum of sinc functions with exponential weights, and Abrarov et al. 8] exploited the Fourier expansion of the Gauss function. In contrast to these quite sophisticated approaches, Jiménez-Mier 9 suggested to use a triangular function approximation for the Gaussian; an assessment of this and similar "triangular Gauss approximations" has been given recently by Schreier [10].

There appears to be no unique definition of "closed-form" [11, however, this term is frequently used, e.g., to motivate numerical quadrature [12, 13. Here we designate expressions containing only (a finite number of) elementary functions as closed-form, i.e. we exclude expressions requiring case distinction (equivalent to conditional branches in the corresponding computer implementation). Using this convention there are only a few Voigt function algorithms satisfying this "closed-form criterion". For example, algorithms based on series and asymptotic expansions obviously require IF branches to distinguish between small and large arguments $x, y$. Continued fraction expansions are in principle valid for the entire range of arguments $(y>0)$, however, for efficiency reasons they are often combined with other methods for small and large arguments [e.g. 14. Rational approximations are known for efficient and accurate complex error function algorithms 15-18, but only the Hui et al. 15] and Weideman [18] implementation provide a unique function for the entire domain. (Note, however, that the Hui et al. algorithm fails for small argument $y<1$, [e.g. 6, 19].) Numerical quadrature of the convolution integral can also be used [1, 20 29], but algorithms based on Gauss-Hermite quadrature [1, 21, 28, 29] are confined to large arguments only and are therefore not considered as closed-form.

In this note we examine closed-form expressions for the Voigt function based on rational function representations of the Gaussian. In the next section we briefly review the definitions and describe our methodology, and we present the resulting approximations along with comparisons with an accurate Voigt function reference in section 3. The codes have been implemented in Python and functions from Scientific Python (scipy.org) have been used as a reference. After a discussion in section 4, a summary and conclusions are given in the final section 5. For this assessment one should note that the Lorentz to Gauss width ratio $y$ encompasses about ten orders of magnitude for Earth atmospheric 
spectroscopy [cf., e.g. 19]).

\section{Theory}

\subsection{The Gaussian function}

Rational functions are quotients of a polynomial of degree $m$ in the numerator and a degree $n$ polynomial in the denominator. Rational approximations $G_{m n}$ for the Gaussian $G(t)=\exp \left(-t^{2}\right)$ can be easily obtained from rational approximations for the exponential function [e.g. 4, section 17.12].

$$
\begin{aligned}
G_{01}(t) & =\frac{1}{1+t^{2}} & G_{11}(t) & =\frac{2-t^{2}}{2+t^{2}} \\
G_{21}(t) & =\frac{6-4 t^{2}+t^{4}}{6+2 t^{2}} & G_{31}(t) & =\frac{4-3 t^{2}+t^{4}+4 t^{6}}{4+t^{2}} \\
G_{02}(t) & =\frac{2}{2+2 t^{2}+t^{4}} & G_{12}(t) & =\frac{6-2 t^{2}}{6+4 t^{2}+t^{4}} \\
G_{22}(t) & =\frac{12-6 t^{2}+t^{4}}{12+6 t^{2}+t^{4}} & G_{32}(t) & =\frac{60-36 t^{2}+9 t^{4}-t^{6}}{60+24 t^{2}+3 t^{4}} \\
G_{03}(t) & =\frac{6}{6+6 t^{2}+3 t^{4}+t^{6}} & G_{13}(t) & =\frac{4-t^{2}}{4+3 t^{2}+t^{4}+4 t^{6}} \\
G_{23}(t) & =\frac{60-24 t^{2}+3 t^{4}}{60+36 t^{2}+9 t^{4}+t^{6}} & G_{33}(t) & =\frac{120-60 t^{2}+12 t^{4}-t^{6}}{120+60 t^{2}+12 t^{4}+t^{6}}
\end{aligned}
$$

For $n=0$ the rational approximations $G_{m 0}$ are identical to the Taylor expansion of degree $m$. A comparison of these rational approximations is given in Fig. 1 Functions with a low degree polynomial in the numerator and denominator are reasonably good only for small $|t| \leq 1$. All approximations with a quadratic function in the denominator $\left(G_{m 1}\right)$ strongly diverge for large $|t| \geq 1$. Only a few approximations, in particular $G_{22}, G_{33}$ and possibly $G_{32}$ appear to be promising candidates and will be examined in the following section.

\subsection{The Voigt function}

The Voigt function (normalized to $\sqrt{\pi}$ ) is defined by

$$
K(x, y)=\frac{y}{\pi} \int_{-\infty}^{\infty} \frac{\mathrm{e}^{-t^{2}}}{(x-t)^{2}+y^{2}} \mathrm{~d} t
$$

where $x$ is a measure of the distance to the center peak, and $y$ is essentially the ratio of the Lorentzian and Gaussian width. At the line center $x=0$ the Voigt function can be expressed as the exponentially scaled complementary error function

$$
K(0, y)=\exp \left(y^{2}\right)(1-\operatorname{erf}(y))=\exp \left(y^{2}\right) \operatorname{erfc}(y) .
$$

The Voigt function is symmetric, i.e. $K(-x, y)=K(x, y)$ and essentially reduces to the Lorentz function for large $|x+\mathrm{i} y|$. 

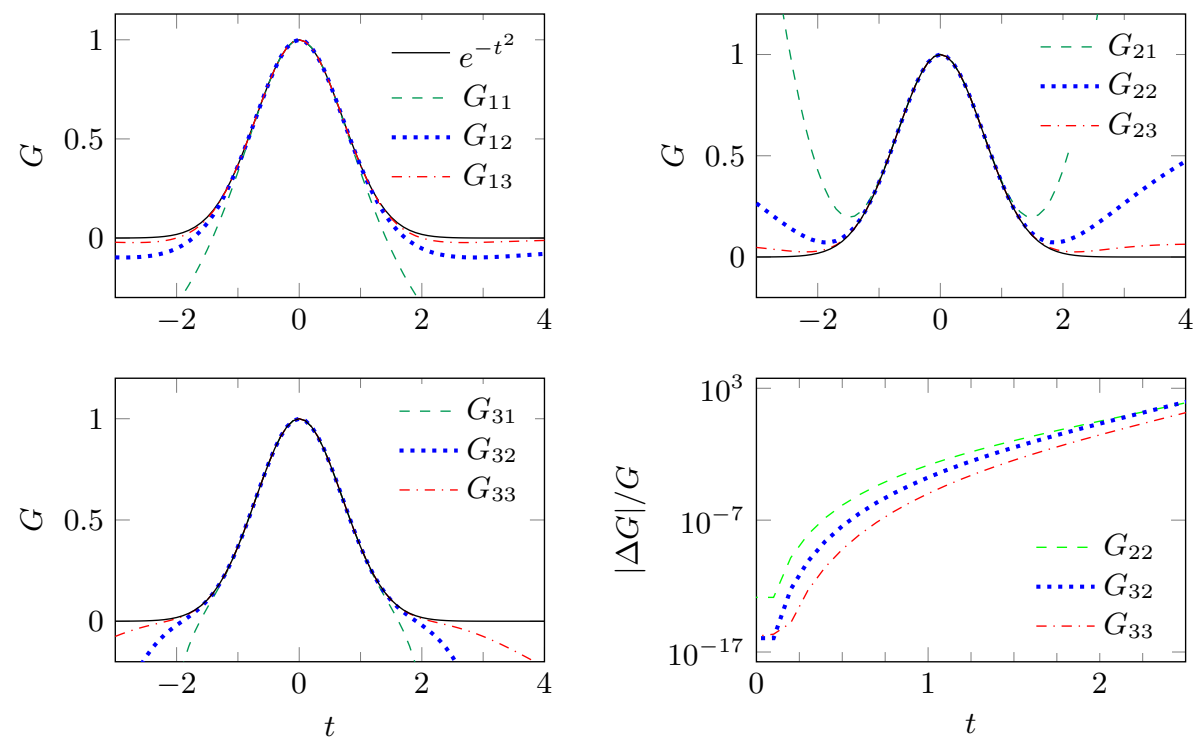

Figure 1: The rational approximations to the Gauss function; Bottom right: The relative error $\Delta G_{m n}(t) / G(t) \equiv\left|G_{m n}(t)-G(t)\right| / G(t)$.

\subsection{Partial fractions}

Inserting any rational approximation for the Gaussian in the integral of (1) transforms the integrand into another rational function. The solution of such integrals relies on partial fractions: first the (real or complex) roots of the denominator polynomial are determined to factorize the denominator. Then the rational function can be rewritten as a sum of rational functions with lowerdegree polynomials in the denominator; in the present context the denominator polynomials are usually quadratic or linear functions of $t$.

The product of the Lorentzian with rational approximations for the Gaussian will often lead to the quotient of a linear function and the product of two quadratic functions that can be transformed according to

$$
\frac{\alpha t+\beta}{\left(t^{2}+p t+q\right)\left(t^{2}-2 x t+s\right)}=\frac{A t+B}{t^{2}+p t+q}+\frac{C t+D}{t^{2}-2 x t+s}
$$

with $s \equiv x^{2}+y^{2}$ and

$$
\begin{aligned}
A=-C & =(\alpha(s-q)+\beta(p-2 x)) / N \\
B & =\left(2 x(\beta p-\alpha q)+\beta s-\alpha p q-\beta q+\beta p^{2}\right) / N \\
D & =\left(4 \beta x^{2}+2 x(\alpha s+\beta p)+\alpha p s-\beta s+\beta q\right) / N
\end{aligned}
$$

and the common denominator

$$
\begin{aligned}
N & =4 q x^{2}+2 p x(s+q)+s^{2}+\left(p^{2}-2 q\right) s+q^{2} \\
& =4 q x^{2}+2 p x(s+q)+p^{2} s+(s-q)^{2} .
\end{aligned}
$$


Note that the denominator is independent of $\alpha$ and $\beta$.

\subsection{The error function}

Rational approximations for the Gauss function can also be used to evaluate the error function defined as

$$
\operatorname{erf}(x)=\frac{2}{\sqrt{\pi}} \int_{0}^{x} \mathrm{e}^{-t^{2}} \mathrm{~d} t \quad \text { with } \quad \operatorname{erf}(\infty)=1 .
$$

First, this will give some insight into the quality of the rational approximation (in addition to the visual inspection in Fig. 11. Furthermore, evaluation of the

integral $\int_{0}^{T} G_{m n}(t) \mathrm{d} t$ will allow the correct normalization of the approximation by proper selection of the upper integral limit. Note that these rational approximations could also be used to construct closed-form expressions for the Dawson function defined by $F(x)=\exp \left(-x^{2}\right) \int_{0}^{x} \exp \left(t^{2}\right) \mathrm{d} t[2]$.

\section{Results}

\subsection{The 2/2 approximation}

The denominator of the $G_{22}$ rational approximation has four complex roots $\pm \sqrt{ \pm \mathrm{i} \sqrt{3}-3}$, but for the following the factorization as $t^{4}+6 t^{2}+12=\left(t^{2}+\right.$ $p t+q)\left(t^{2}-p t+q\right)$ with $q=\sqrt{12}$ and $p=\sqrt{2 q-6}=\sqrt{4 \sqrt{3}-6}$ is sufficient. Using the partial fractionation of $G_{22}$

$$
G_{22}(t)=1+\frac{6 t / p}{t^{2}+p t+q}-\frac{6 t / p}{t^{2}-p t+q}
$$

allows to approximate the error function

$$
\begin{aligned}
\operatorname{erf}_{22}(x) \equiv & \frac{2}{\sqrt{\pi}} \int_{0}^{x} G_{22}(t) \mathrm{d} t \\
= & \frac{2}{\sqrt{\pi}}\left[x+\frac{3}{p}\left(\ln \left(x^{2}+p x+q\right)-\ln \left(x^{2}-p x+q\right)\right)\right. \\
& \left.-\frac{6}{\sqrt{\Delta}}\left(\arctan \frac{2 x+p}{\sqrt{\Delta}}+\arctan \frac{2 x-p}{\sqrt{\Delta}}\right)\right]
\end{aligned}
$$

with $\Delta=4 q-p^{2}=4 \sqrt{3}+6$. For large $x$ the sum of the two arctan's levelsoff at $\pi$, whereas the difference of the logarithms tends to zero, so asymptotically this approximation is proportional to $x$ instead of a constant. Fig. 2 compares this approximation with the "exact" function values evaluated by the scipy.special.erf algorithm, indicating a relative error less than $1 \%$ for $x<T$. Using Brent's method (in the scipy.optimize . brentq implementation) the integral upper limit is determined to $T=1.81762647$ such that $\operatorname{erf}_{22}(T)=1$. 

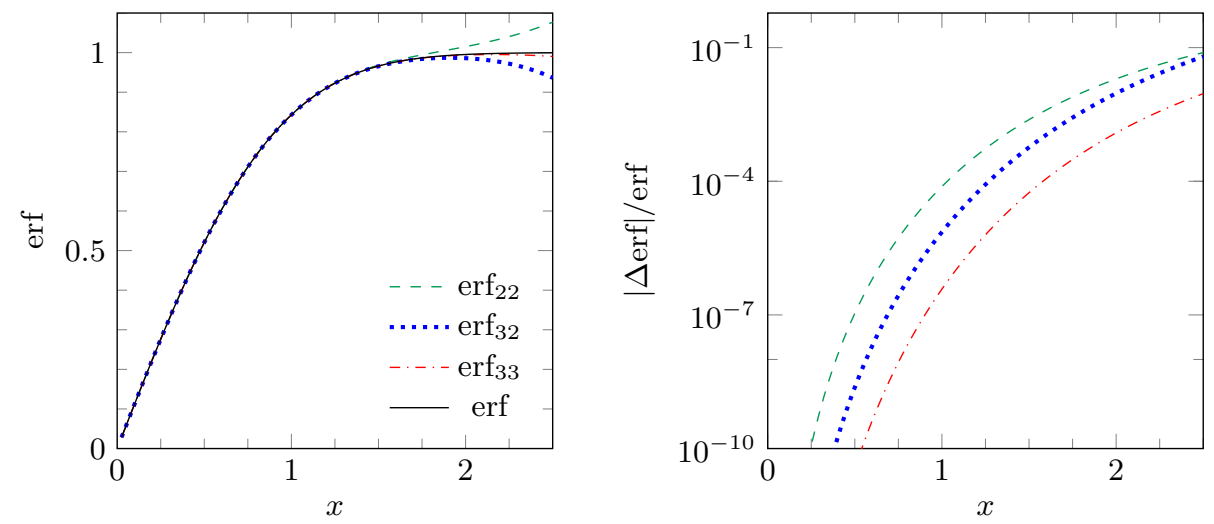

Figure 2: Left: Approximations to the error function based on rational approximations for the Gaussian; Right: The relative error $\mid \Delta$ erf $\mid /$ erf.

The rational approximation (7) for the Gaussian can now be used to evaluate the Voigt integral (1) with the integral bounds $\pm T$ to ensure a proper normalization

$$
K_{22}(x, y)=\frac{y}{\pi} \int_{-T}^{T} \frac{G_{22}(t) \mathrm{d} t}{t^{2}-2 x t+x^{2}+y^{2}} .
$$

This gives two quotients similar to those on the left hand side of (3) (with $\beta=0$ ), and the decomposition leads to four integrals

$$
\begin{aligned}
I(T ; a, b, c, d) \equiv & \int_{-T}^{T} \frac{a t+b}{t^{2}+c t+d} \mathrm{~d} t \\
= & \frac{a}{2}\left[\log \left(T^{2}+c T+d\right)-\log \left(T^{2}-c T+d\right)\right] \\
& +\frac{2 b-c a}{\sqrt{4 d-c^{2}}}\left[\arctan \frac{2 T+c}{\sqrt{4 d-c^{2}}}-\arctan \frac{-2 T+c}{\sqrt{4 d-c^{2}}}\right] .
\end{aligned}
$$

The constant term in (7) leads to the integral of a Lorentz function (the first term in the next equation). This results in the Voigt function approximation

$$
\begin{aligned}
K_{22}(x, y)=\frac{1}{\pi}[ & \left.\arctan \frac{T-x}{y}+\arctan \frac{T+x}{y}\right] \\
+ & \frac{6 y}{p \pi}\left[I\left(T ; A_{+}, B_{+},+p, q\right)+I\left(T ;-A_{+}, D_{+},-2 x, x^{2}+y^{2}\right)\right. \\
& \left.\quad-I\left(T ; A_{-}, B_{-},-p, q\right)-I\left(T ;-A_{-}, D_{-},-2 x, x^{2}+y^{2}\right)\right]
\end{aligned}
$$

where $A_{ \pm}, B_{ \pm}$and $D_{ \pm}$are the coefficients of (4) and (5) corresponding to $t^{2} \pm p t+q$ in (3). Note that $A_{ \pm}, B_{ \pm}$, and $D_{ \pm}$are themselve functions of $x$ and 
$y$. Inserting 10 leads to

$$
\begin{aligned}
K_{22}(x, y)= & \frac{3 y}{p \pi}\left[\left(A_{+}+A_{-}\right) \log \left(\frac{T^{2}+p T+q}{T^{2}-p T+q}\right)-\left(A_{+}-A_{-}\right) \log \left(\frac{T^{2}-2 x T+q}{T^{2}+2 x T+q}\right)\right] \\
& +\frac{6 y}{p \sigma \pi}\left(2\left(B_{+}-B_{-}\right)-p\left(A_{+}+A_{-}\right)\right)\left(\arctan \frac{2 T+p}{\sigma}+\arctan \frac{2 T-p}{\sigma}\right) \\
& +\left(1+\frac{6}{p}\left(D_{+}-D_{-}-x\left(A_{+}-A_{-}\right)\right)\right)\left(\arctan \frac{T+x}{y}+\arctan \frac{T-x}{y}\right) / \pi
\end{aligned}
$$

with $\sigma=\sqrt{\Delta}=\sqrt{4 q-p^{2}} \approx 3.6$. The difference $A_{+}-A_{-}$is antisymmetric in $x$, whereas the sum $A_{+}+A_{-}$as well as the differences $B_{+}-B_{-}$and $D_{+}-D_{-}$ are symmetric in $x$, and accordingly $K_{22}(x, y)$ is an even function of $x$.

In Fig. 3 the quality of this approximation is displayed using the Weideman [18] rational approximation (" $a$-expansion" with 32 terms) as a reference. For $y \geq 1$ the $K_{22}$ approximation appears to be quite good, hardly discernible from the reference, and the relative difference shown on the right indicates an error less than $10^{-2}$. However, for smaller $y$ discrepancies can be seen around $x=T$ that are confirmed by large relative differences up to about $10^{-1}$. Inspection of the individual terms in (13) indicates that $\arctan (T-x) / y$ resulting from the integration of the Lorentzian suddently drops from $+\pi / 2$ to $-\pi / 2$ at $x=T$, especially for small $y$, whereas the other terms are much smoother. (Similar deviations can also be observed in the Voigt function representations resulting from the triangular approximation for the Gaussian, see Fig. 2 of Schreier [10].)

As a further test of the correct evaluation of the integral (9) it has been recalculated numerically using an adaptive quadrature routine of QUADPACK [30] (using scipy.integrate.quad). For $y=0.01$ Fig. 3 demonstrates that the failure around $x \approx T$ is related to the rational approximation used for the Gauss function, whereas the numerically computed integral (1) also shown in the figure clearly resembles the values provided by the reference implementation.

\subsection{The 3/2 approximation}

Fig. 1 indicates that the $3 / 2$ rational function might also allow a reasonable approximation for the Voigt function. Partial fractionation leads to

$$
G_{32}(t)=\frac{1}{3}\left[17-t^{2}-\frac{\alpha t+\beta}{t^{2}+p t+q}+\frac{\alpha t-\beta}{t^{2}-p t+q}\right]
$$

with

$$
\alpha=\frac{140-76 q}{p q} \quad \text { and } \quad \beta=\frac{140}{q}
$$

and

$$
q=\sqrt{20} \text { and } p=\sqrt{2 q-8}
$$




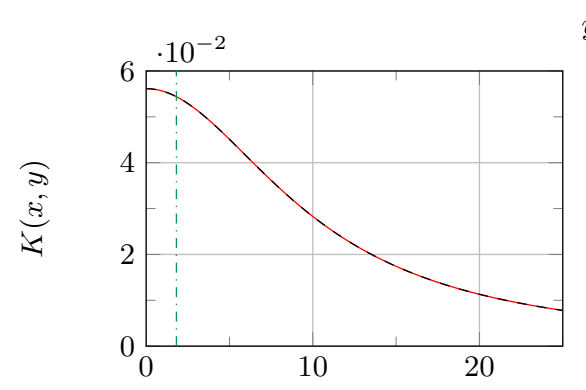

$y=10$
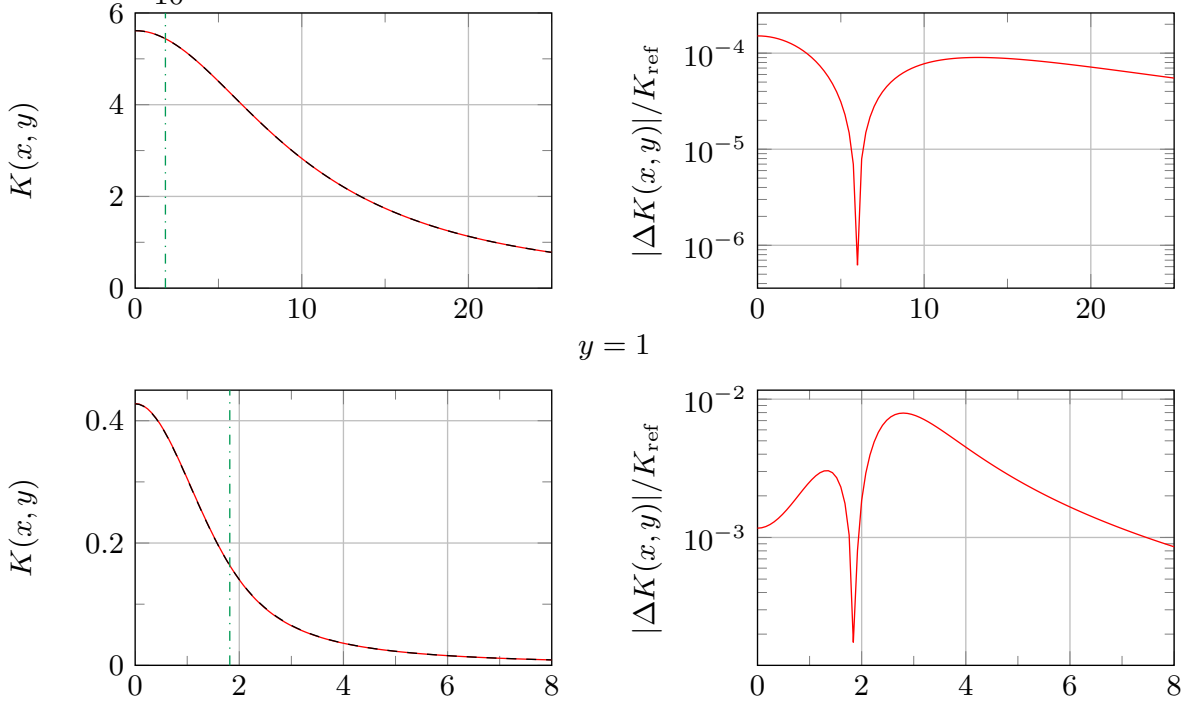

$y=1$
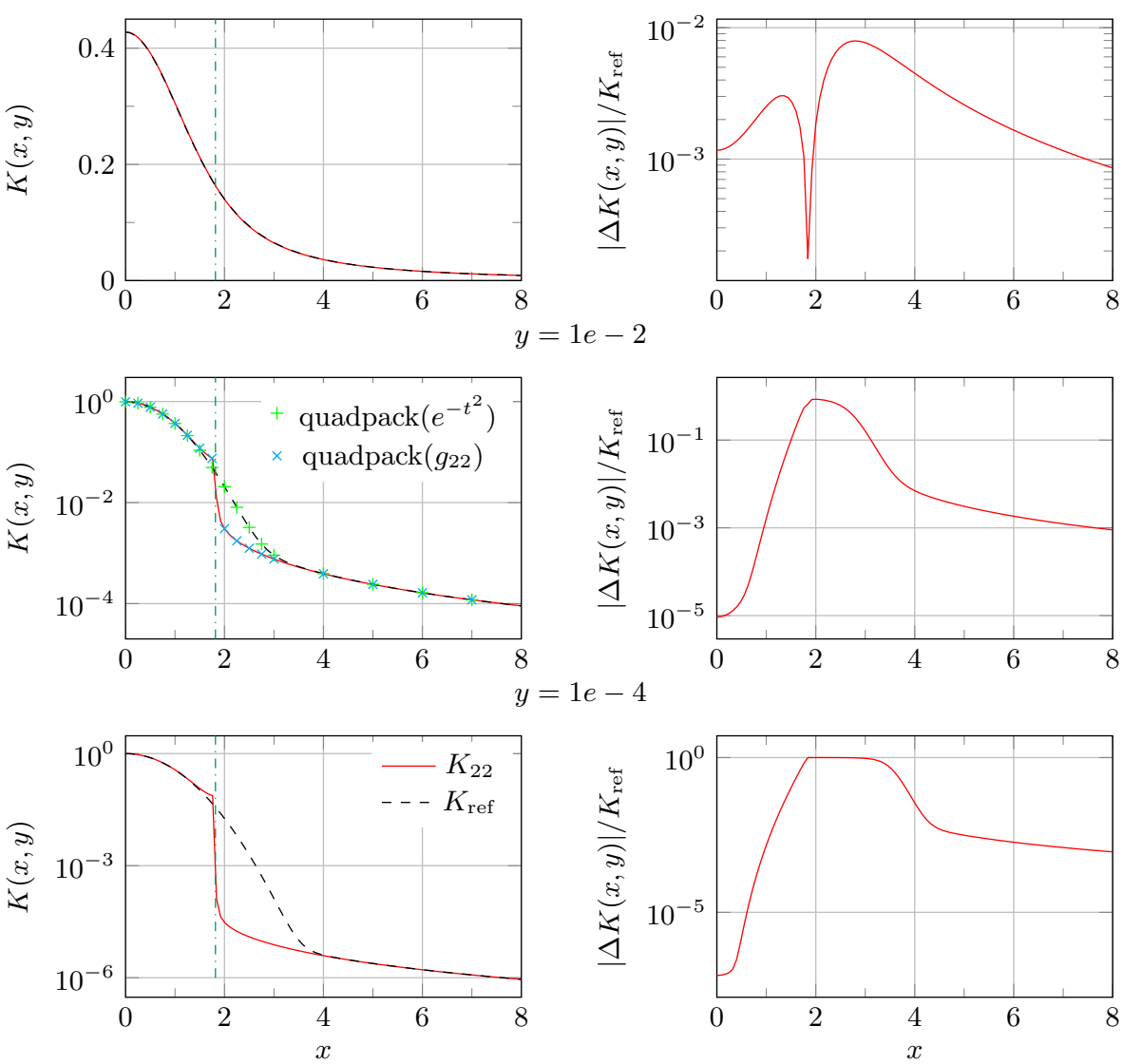

Figure 3: Left: Approximation to the Voigt function based on the 2/2 rational approximation for the Gaussian; ' $x$ ' and '+' are integral values evaluated numerically by QUADPACK for a subset of $x$ values and $y=0.01$. The green dash-dotted line indicates the integration limit $T$. Right: The relative error $\left|K_{22}-K_{\text {ref }}\right| / K_{\text {ref. }}$. Note the extended $x$ axis in the top row and the logarithmic $K$ axis for small $y$. 
Using this approximation the integral (6) defining the error function can be evaluated analytically,

$$
\begin{aligned}
\operatorname{erf}_{32}(x) \equiv \frac{2}{\sqrt{\pi}} \int_{0}^{x} G_{32}(t) \mathrm{d} t \\
=\frac{2}{3 \sqrt{\pi}}\left[17 x-\frac{x^{3}}{3}+\frac{\alpha}{2}\left[\ln \left(x^{2}-p x+q\right)-\ln \left(x^{2}+p x+q\right)\right]\right. \\
\left.\quad+\frac{\alpha p-2 \beta}{\sqrt{2 q+8}}\left(\arctan \frac{2 x+p}{\sqrt{2 q+8}}+\arctan \frac{2 x-p}{\sqrt{2 q+8}}\right)\right]
\end{aligned}
$$

Compared to (8) there is a new term proportional to the cube of $x$, but otherwise it is quite similar except for the constants. Fig. 2 indicates that this approximation has a maximum at $T=1.90727351$ with a function value of $\operatorname{erf}_{32}(T)=0.98696416$ determined with scipy.optimize.minimize. To ensure a proper normalization of the Gauss approximation a scaled function $\tilde{G}_{32} \equiv$ $G_{32} / \operatorname{erf}_{32}(T)$ is defined and can be used to compute an approximation for the Voigt function.

Inserting (14) into (1) gives

$$
\begin{aligned}
K_{32}(x, y)= & \frac{y}{\pi} \int_{-T}^{T} \frac{G_{32}(t) \mathrm{d} t}{t^{2}-2 x t+x^{2}+y^{2}} \\
= & \frac{y}{3 \pi} \int_{-T}^{T} \frac{\left(17-t^{2}\right) \mathrm{d} t}{t^{2}-2 x t+x^{2}+y^{2}} \\
+ & \frac{y}{3 \pi}\left[\int_{-T}^{T} \frac{(\alpha t-\beta) \mathrm{d} t}{\left(t^{2}-p t+q\right)\left(t^{2}-2 x t+x^{2}+y^{2}\right)}\right. \\
& \left.\quad-\int_{-T}^{T} \frac{(\alpha t+\beta) \mathrm{d} t}{\left(t^{2}+p t+q\right)\left(t^{2}-2 x t+x^{2}+y^{2}\right)}\right]
\end{aligned}
$$

The first integral (with $s \equiv x^{2}+y^{2}$ ) gives

$$
\begin{aligned}
I_{1} & \equiv \int_{-T}^{T} \frac{\left(17-t^{2}\right) \mathrm{d} t}{t^{2}-2 x t+s} \\
& =\frac{17-x^{2}+y^{2}}{y}\left[\arctan \frac{T-x}{y}+\arctan \frac{T+x}{y}\right]-2 T+x \ln \frac{T^{2}+2 x T+s}{T^{2}-2 x T+s}
\end{aligned}
$$

The second and third integrals are evaluated as described in the previous subsection using the partial fractions of (3), resulting in the Voigt function approximation

$$
\begin{aligned}
K_{32}(x, y)=\frac{y}{3 \pi}\left(I_{1}\right. & +I\left(T ; A_{-}, B_{-},-p, q\right)+I\left(T ;-A_{-}, D_{-},-2 x, s\right) \\
& \left.-I\left(T ; A_{+}, B_{+},+p, q\right)-I\left(T ;-A_{+}, D_{+},-2 x, s\right)\right)
\end{aligned}
$$




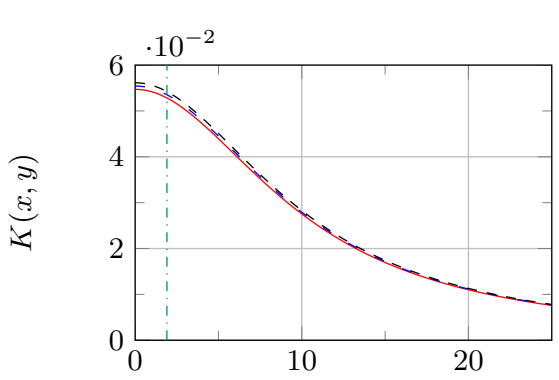

$y=10$

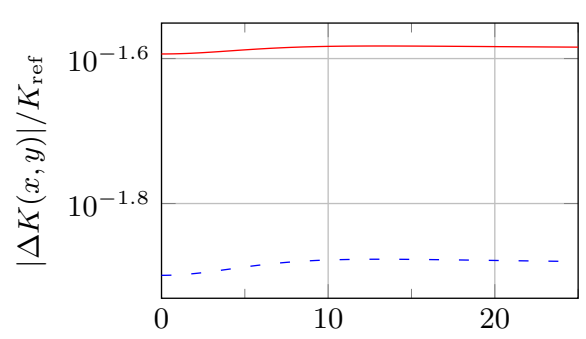

$y=1$
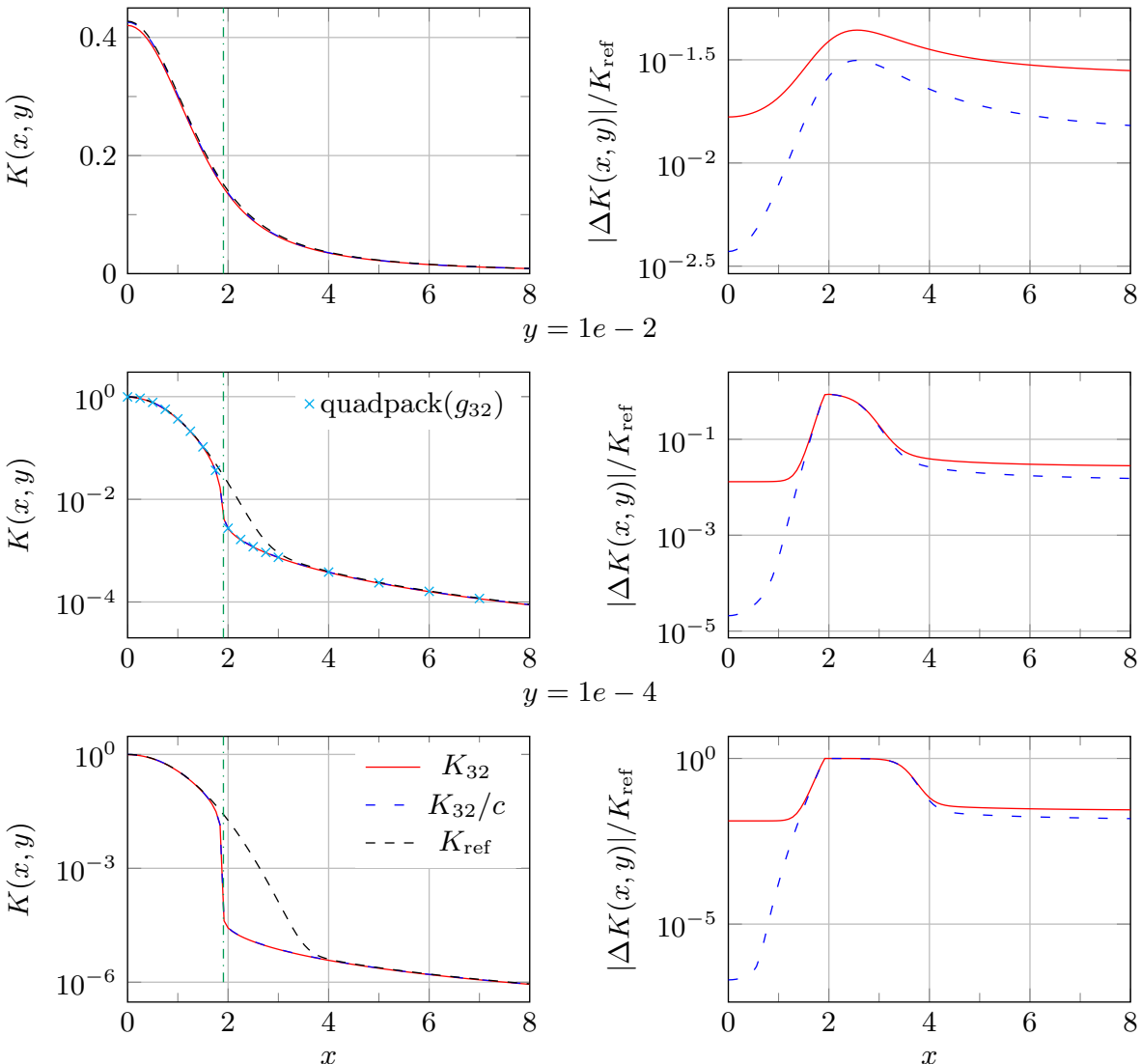

Figure 4: Left: Approximation to the Voigt function based on the 3/2 rational approximation for the Gaussian (red: without normalization; blue, long-dashed: with normalization, 'x': numerical quadrature result). Right: The relative error $\left|K_{32}-K_{\text {ref }}\right| / K_{\text {ref }}$. 
Fig. 4 shows that the approximation based on the $3 / 2$ rational function for the Gaussian without normalization is worse than the $2 / 2$ approximation, indicating the importance of a properly normalized Gauss approximation. However, for small $x$ the scaled approximation $K_{32} / \operatorname{erf}_{32}(T)$ based on $\tilde{G}_{32}$ is significantly better, whereas for larger $x$ both scaled and unscaled approximations behave similarly with errors of some percent. For small $y<1$ this approximation shows the same problem as the $K_{22}$ approximation, i.e. a strong, almost stepwise decrease around $T \approx 1.9$ due to the $\arctan (T-x) / y$ term. As for the $K_{22}$ approximation above, the closed-form expression $(18)$ has been checked for $y=0.01$ and selected values of $x$ using QUADPACK's numerical quadrature of (16).

\subsection{The 3/3 approximation}

Seen as a cubic polynomial of $\tau \equiv t^{2}$, the denominator of $G_{33}$ has one real root $\tau_{r}=u+v-4$ and two complex roots, and $G_{33}$ can be expressed as

$$
\begin{aligned}
G_{33}(t) & =\frac{24 t^{4}+240}{120+60 t^{2}+12 t^{4}+t^{6}}-1 \\
& =\frac{\gamma}{t^{2}+r}+\frac{\alpha t+\beta}{t^{2}+p t+q}-\frac{\alpha t-\beta}{t^{2}-p t+q}-1
\end{aligned}
$$

where

$$
\begin{aligned}
r & =4-u-v \\
u & =\sqrt[3]{4 \sqrt{5}-4} \\
v & =\sqrt[3]{4 \sqrt{5}+4}=-4 / u
\end{aligned}
$$

The second and third denominators are defined by the constants

$$
q=\sqrt{16+4(u+v)+u^{2}-u v+v^{2}} \text { and } p=\sqrt{2 q-8-u-v}
$$

and the constants in the numerators are

$$
\begin{aligned}
\gamma & =\left(24 r^{2}+240\right) / N \\
\alpha & \left.=-12\left(p^{2} q r-10\right)+(q-r)\left(q^{2}+10\right)\right) /(p q N) \\
\beta & =\left(12\left(10-q^{2}\right) r-240 q+120 p^{2}\right) /(q N)
\end{aligned}
$$

with the denominator $N=(r-q)^{2}+p^{2} r$.

Defining $\Delta=4 q-p^{2}$ similar to above results in the approximation for the error function

$$
\begin{aligned}
& \operatorname{erf}_{33}(x) \equiv \frac{2}{\sqrt{\pi}}[ \frac{\gamma}{\sqrt{r}} \arctan \frac{x}{\sqrt{r}}+\frac{\alpha}{2}\left(\log \left(x^{2}+p x+q\right)-\log \left(x^{2}-p x+q\right)\right) \\
&\left.\frac{2 \beta-p \alpha}{\sqrt{\Delta}}\left(\arctan \frac{2 x+p}{\sqrt{\Delta}}+\arctan \frac{2 x-p}{\sqrt{\Delta}}\right)-x\right]
\end{aligned}
$$




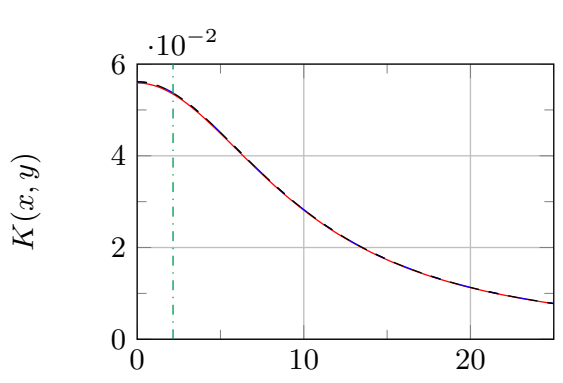

$y=10$
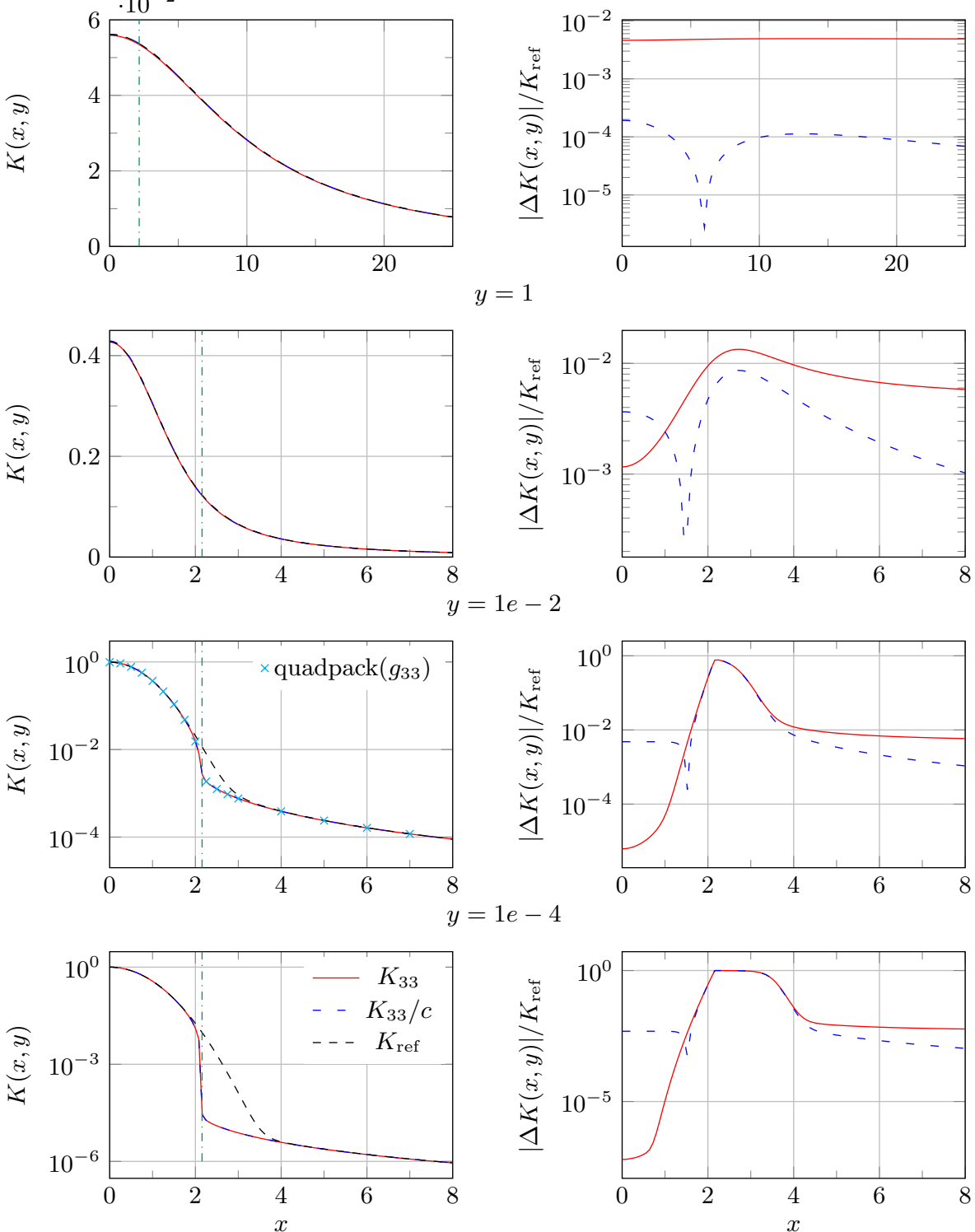

Figure 5: Left: Approximation to the Voigt function based on the 3/3 rational approximation for the Gaussian; (red: without normalization; blue, long-dashed: with normalization, 'x': numerical quadrature result). Right: The relative error $\left|K_{33}-K_{\text {ref }}\right| / K_{\text {ref }}$. 
Similar to $\operatorname{erf}_{32}$ this approximation diverges for large $x$, i.e. $\operatorname{erf}_{33} \rightarrow-\infty$. Fig. 2 also depicts this approximation, which is clearly better than the approximations presented in the previous subsections. However, similar to (15) (but unlike (8)), it has a maximum at $T=2.15503361$ slightly smaller than one.

Inserting the rational function 19 into the definition of the Voigt function (1) leads to integrands similar to above. The first, second, and third term lead to integrands of the form (3), and the last term leads to an integral of the Lorentz function, and finally

$$
\begin{gathered}
K_{33}=\frac{y}{\pi}\left[\arctan \frac{T-x}{y}+\arctan \frac{T+x}{y}\right]+ \\
+\left[I\left(T ; A_{+}, B_{+},+p, q\right)+I\left(T ;-A_{+}, D_{+},-2 x, x^{2}+y^{2}\right)\right. \\
\left.I\left(T ; A_{-}, B_{-},-p, q\right)+I\left(T ;-A_{-}, D_{-},-2 x, x^{2}+y^{2}\right)\right] \\
\left.I\left(T ; A_{g}, B_{g}, 0, r\right)+I\left(T ;-A_{g}, D_{g},-2 x, x^{2}+y^{2}\right)\right]
\end{gathered}
$$

where $A_{g}, B_{g}$ and $D_{g}$ are the coefficients as in (4) for the first term in 19$]$.

Fig. 5 reveals that this approximation, compared to the Weideman [18] reference, is slightly better for $y=1$, both without and with normalization. Interestingly, the unnormalized $K_{33}$ is better for small $y$ than the normalized $\tilde{K}_{33} \equiv K_{33} / \operatorname{erf}_{33}(T)$ in contrast to the $3 / 2$ approximation presented above. However, similar to the previous approximation $K_{33}$ and $\tilde{K}_{33}$ strongly deviate from the reference for $x$ near the integration limit $T$.

\subsection{Computational efficiency}

Similar to the closed-form expressions resulting from a triangle-approximation for the Gauss function (see Eq. (5) of Schreier [10]), the expressions (13), (18), and (26) presented here contain logarithms, inverse tangents, and rational functions. The timing benchmark tests performed then have shown that these approximations are about a factor 4 or 5 slower than the Humlíček-Weideman combination of rational approximations [19. Although some of the logarithms and arctan's in (13), (18), and (26) are independent of $x$ and $y$, the expressions are more complicated now, and the evaluation of the Voigt function is likely to be even more expensive.

To further quantify this estimate, the approximation 13 has also been implemented in Fortran (2008) and performance tests have been conducted similar to those documented in [19, i.e. molecular absorption cross sections of $\mathrm{HNO}_{3}$ have been evaluated for a sequence of 13 atmospheric levels and spectroscopic line data from the HITRAN 2008 database [31] in the microwave regime (wavenumbers $16-17 \mathrm{~cm}^{-1}$ with about 17000 lines from $6-27 \mathrm{~cm}^{-1}$ contributing. In this example, the Voigt parameter $y$ (proportional to pressure) decreases from approximately $10^{4}$ at bottom to $10^{-4}$ at top of atmosphere.

Table 1 confirms these expectations, i.e. the Voigt function approximation derived with a rational approximation for the Gaussian is a factor 16 to 22 slower 


\begin{tabular}{cccc} 
version & optimization & Eq. $[13)$ & Ref. [19] \\
\hline 4.8 .1 & O0 & 285.9 & 13.16 \\
4.8 .1 & O3 & 78.73 & 4.370 \\
6.2 .1 & O0 & 279.5 & 12.89 \\
6.2 .1 & O3 & 78.94 & 5.078 \\
\hline
\end{tabular}

Table 1: Run time (in nanoseconds) per function evaluation (i.e., number of lines times number of wavenumber grid points) for the 13 compared to the Humlícek.Weideman combination. Different versions of the gfortran compiler and optimization levels are used.

than the Humlíček-Weideman approximation for the complex error function. Note that the approximations of [10 require two (or three) logarithm calls and three inverse tangent calls for a single $x$ value (corresponding to wavenumber), but there is no division of any $x$ dependent quantity. In contrast, the evaluation of 13 involves seven $x$ dependent divisions in addition to one $x$ dependent logarithm and two $x$ dependent arctan's (the other logarithms and arctan's are independent of $x$ and $y$ ).

\section{Discussion}

According to Goedecker and Hoisie [32, "the calculation of special functions, such as divisions, square roots, exponentials and logarithms requires anywhere from a few dozen cycles up to hundreds of cycles. ...... these calculations have to be decomposed into a sequence of elementary instructions such as multiplies and adds." (See also subsection 5.5.6 in [13.) First note the inclusion of division in this list of "special functions". Note also that with special computer hardware (e.g. field-programmable gate arrays - FPGA) the penalty of divisions can be overcome. Rational approximations as Humlíček [17] or Weideman [18] require only a single division in addition to additions and multiplications. The large number of "special function" calls in the approximations developed here will therefore lead to a large computational burden.

For high-resolution infrared and microwave atmospheric spectroscopy the line-by-line modeling of molecular cross sections constitutes a significant computational challenge. Million to billion evaluations of the Voigt function are not uncommon for radiative transfer calculations. This is especially demanding in the context of operational data processing of remote sensing missions; for example, the IASI instruments aboard the MetOp A and B meteorological satellites each deliver 1.3 million spectra per day [33]. Moreover, for some applications (e.g. high temperatures) the "standard" HITRAN 34] or GEISA [35] databases with several million lines each are insufficient and more complete databases [36, 37. with billions of lines have to be considered.

Rational functions with higher degree of the numerator and/or denominator polynomials can provide a better approximation of the Gauss function (at least for small $x$ where the contribution to the integral is significant) and it is therefore tempting to use these to evaluate the Voigt convolution integral. However, the 


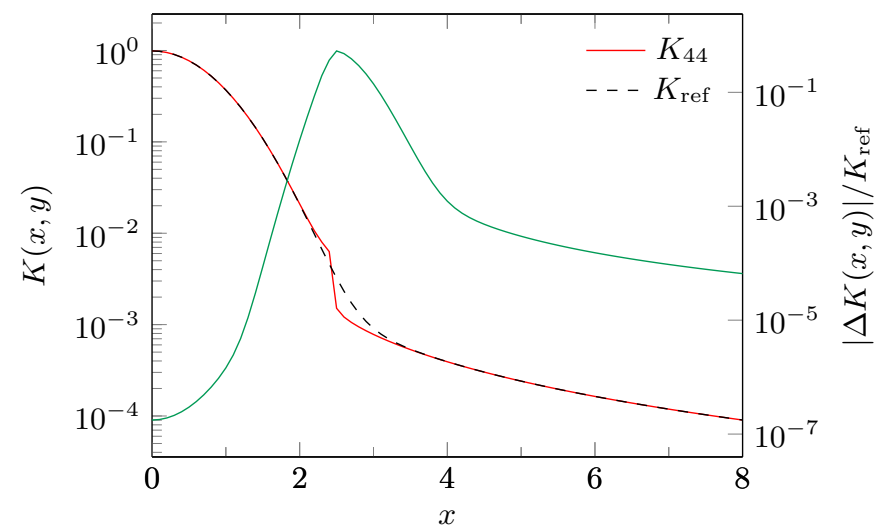

Figure 6: Approximation to the Voigt function based on the 4/4 rational approximation for the Gaussian and its relative error (right axis) for $y=0.01$.

resulting approximations will likely involve $\arctan (x-T) / y$ terms similar to above (where $T$ depends on the particular rational function) that give rise to the large discrepancies around $x \approx T$ for small $y$. This is clearly confirmed by the approximation based on the 4/4 rational function approximation of the Gaussian shown in Fig. 6. Moreover, the determination of the partial fractions becomes increasingly expensive, and the computational efficiency of the resulting sum of various logarithms and inverse trigonometric functions is even worse.

The conditional branches required for most modern (efficient and accurate) Voigt and complex error function algorithms can be suboptimal for some programming languages, and a closed-form approximation could be welcome. Algorithms based on direct numerical quadrature of the Voigt integral (1) might be a suitable approach. Matta and Reichel [22] suggested to use trapezoid quadrature; some problems of this algorithm have been addressed by Hunter and Regan 23 and Lether and Wenston [26, and a vectorized version has been provided by Lynas-Gray [27. Finn and Mugglestone [20] used Simpson quadrature to compile a table of Voigt function values (for $y \geq 0.01$ ), exploiting a modified integrand for small $x$ values. Parthasarathi et al. 24] considered the infinite integral (1) difficult for numerical integration and used Newton-Cotes quadrature of the finite integral resulting from the transformation $t=u /(1-u)$. Afonin et al. 25] concluded that a Fast Fourier transform approach is more efficient than an adaptive quadrature procedure. However, accurate function values can only be obtained with a proper sampling of the integrand, i.e. a large number of exponent evaluations and divisions. With Gauss-Hermite quadrature there is no need to compute exponentials, but still it requires several Lorentz function evaluations for a single Voigt function value (Armstrong [1] uses a 20-term Gauss-Hermite quadrature with the original and modified integrand for large and medium sized arguments, respectively). In conclusion, Voigt function algorithms based on numerical quadrature schemes are therefore not expected to be competitive with rational approximations. 


\section{Summary and Conclusions}

Closed-form expressions for the Voigt function have been constructed using rational approximations for the Gauss function that allowed an explicit analytical solution of the integral after appropriate partial fractionation of the integrand. Although the rational approximations for the Gaussian considered here are better than the triangular approximations considered previously [10] the resulting approximations for the Voigt function completely fail for $x \approx 2$ and small $y \ll 1$.

Our conclusions now are therefore similar to the conclusions given in Schreier [10: Closed-form expressions as presented here might be desirable for certain applications, but their quality is limited to only a small $y$ interval. When closedform expressions are really necessary, approximations based on combinations of Lorentz and Gauss functions [see 10, for references] might be a reasonable alternative. (An assessment of the quality of these representations is beyond the scope of this work.) In all other cases approximations based on modern state-of-the-art numerical methods, e.g. rational approximations as discussed in Humlíček [17, Weideman [18, and Schreier [19] are recommended.

\section{Acknowledgments}

First I would like to thank the two reviewers of Schreier [10] for the very positive feedback that encouraged me to continue with these kinds of approximations. My son Lukas brought my attention back to partial fractions in a high school seminar work some years ago. Financial support by the Deutsche Forschungsgemeinschaft — DFG (project SCHR 1125/3-1) is gratefully acknowledged.

\section{References}

[1] B.H. Armstrong. Spectrum line profiles: The Voigt function. J. Quant. Spectrosc. E Radiat. Transfer, 7:61-88, 1967. doi: 10.1016/0022-4073(67)90057-X.

[2] F.W.J. Olver, D.W. Lozier, R.F. Boisvert, and C.W. Clark, editors. NIST Handbook of Mathematical Functions. Cambridge University Press, New York, NY. Print companion to 3 .

[3] DLMF. NIST Digital Library of Mathematical Functions. National Institute of Standards and Technology. URL http://dlmf .nist.gov/ Online companion to 2 .

[4] Keith B. Oldham, Jan Myland, and Jerome Spanier. An Atlas of Functions. Springer, 2009. doi: 10.1007/978-0-387-48807-3.

[5] H. E. Salzer. Formulas for calculating the error function of a complex variable. Math. Tables Aids Compu., 5(34):67-70, 1951.

[6] M.R. Zaghloul and A.N. Ali. Algorithm 916: Computing the Faddeyeva and Voigt functions. ACM Trans. Math. Soft., 38(2):15:1-15:22, January 2011. doi: 10.1145/2049673.2049679. 
[7] G.B. Rybicki. Dawson's integral and the sampling theorem. Computers in Physics, 3(March/April):85-87, 1989.

[8] S.M. Abrarov, B.M. Quine, and R.K. Jagpal. Rapidly convergent series for highaccuracy calculation of the Voigt function. J. Quant. Spectrosc. 8 Radiat. Transfer, 111:372-375, 2010. doi: 10.1016/j.jqsrt.2009.09.005.

[9] J. Jiménez-Mier. An approximation to the plasma dispersion function. J. Quant. Spectrosc. E3 Radiat. Transfer, 70(3):273-284, 2001. doi: 10.1016/S0022-4073(00) 00139-4.

[10] F. Schreier. An assessment of some closed-form expressions for the Voigt function. J. Quant. Spectrosc. \& Radiat. Transfer, 176:1-5, 2016. doi: 10.1016/j.jqsrt.2016. 02.016 .

[11] J.M. Borwein and R.E. Crandall. Closed forms: What they are and why we care. Not. Am. Math. Soc., 60(1):50-65, 2013. doi: 10.1090/noti936.

[12] D. Kahaner, C. Moler, and S. Nash. Numerical Methods and Software. PrenticeHall, Englewood Cliffs, NJ, 1989.

[13] C.W. Ueberhuber. Numerical Computation. Springer, 1997. (2 Volumes).

[14] I.A. Stegun and R. Zucker. Automatic computing methods for special functions. Part IV. Complex error function, Fresnel integrals, and other related functions. Journal of Research of the National Bureau of Standards, 86(6):661-686, 1981.

[15] A.K. Hui, B.H. Armstrong, and A.A. Wray. Rapid computation of the Voigt and complex error functions. J. Quant. Spectrosc. \& Radiat. Transfer, 19:509-516, 1978. doi: 10.1016/0022-4073(78)90019-5.

[16] J. Humlíček. An efficient method for evaluation of the complex probability function: the Voigt function and its derivatives. J. Quant. Spectrosc. \& Radiat. Transfer, 21:309-313, 1979. doi: 10.1016/0022-4073(79)90062-1.

[17] J. Humlíček. Optimized computation of the Voigt and complex probability function. J. Quant. Spectrosc. \& Radiat. Transfer, 27:437-444, 1982 . doi: 10.1016/0022-4073(82)90078-4.

[18] J.A.C. Weideman. Computation of the complex error function. SIAM J. Num. Anal., 31:1497-1518, 1994. doi: 10.1137/0731077.

[19] F. Schreier. Optimized implementations of rational approximations for the Voigt and complex error function. J. Quant. Spectrosc. \& Radiat. Transfer, 112(6): 1010-1025, 2011. doi: 10.1016/j.jqsrt.2010.12.010.

[20] G.D. Finn and D. Mugglestone. Tables of the line broadening function $H(a, v)$. Mon. Not. Roy. Astron. Soc., 129:221-235, 1965. doi: 10.1093/mnras/129.2.221.

[21] S.R. Drayson. Rapid computation of the Voigt profile. J. Quant. Spectrosc. E Radiat. Transfer, 16:611, 1976. doi: 10.1016/0022-4073(76)90029-7.

[22] F. Matta and A. Reichel. Uniform computation of the error function and other related functions. Math. Comp., 25:339-344, 1971. doi: 10.1090/ S0025-5718-1971-0295538-4.

[23] D.B. Hunter and T. Regan. A note on the evaluation of the complementary error function. Math. Comp., 26:539-541, 1972. doi: 10.1090/ S0025-5718-1972-0303685-4.

[24] V. Parthasarathi, S. Ganapathy, and C.A. McDowell. The evaluation and characterization of Voigt lineshape. J. Mol. Structure, 64:29-38, 1980. doi: 10.1016/0022-2860(80)80112-8. 
[25] S.V. Afonin, V.A. Gaponov, and A.G. Gandrin. Comparative analysis of three procedures for calculation of the Voigt profile of a spectral line. J. Appl. Spectros., 41:868-872, 1984. doi: 10.1007/BF00659831.

[26] F.G. Lether and P.R. Wenston. An algorithm of the numerical computation of the Voigt function. Appl. Math. Comp., 35:277-289, 1990. doi: 10.1016/ 0096-3003(90)90047-7.

[27] A.E. Lynas-Gray. VOIGTL - a fast subroutine for Voigt function evaluation on vector processors. Comp. Phys. Comm., 75:135-142, 1993. doi: 10.1016/ 0010-4655(93)90171-8.

[28] Z. Shippony and W.G. Read. A correction to a highly accurate Voigt function algorithm. J. Quant. Spectrosc. \& Radiat. Transfer, 78:255, 2003. doi: 10.1016/ S0022-4073(02)00169-3.

[29] Kendra L. Letchworth and D. Chris Benner. Rapid and accurate calculation of the Voigt function. J. Quant. Spectrosc. \& Radiat. Transfer, 107:173-192, 2007. doi: $10.1016 /$ j.jqsrt.2007.01.052.

[30] R. Piessens, E. de Doncker-Kapenga, C.W. Überhuber, and D.K. Kahaner. QUADPACK - A Subroutine Package for Automatic Integration. Springer, 1983. doi: 10.1007/978-3-642-61786-7.

[31] L.S. Rothman, I.E. Gordon, A. Barbe, D.C. Benner, P.F. Bernath, M. Birk et al. The HITRAN 2008 molecular spectroscopic database. J. Quant. Spectrosc. \& Radiat. Transfer, 110(9-10):533 - 572, 2009. doi: 10.1016/j.jqsrt.2009.02.013.

[32] Stefan Goedecker and Adolfy Hoisie. Performance Optimization of Numerically Intensive Codes. SIAM, Philadelphia, PA, 2001.

[33] C. Clerbaux, A. Boynard, L. Clarisse, M. George, J. Hadji-Lazaro, H. Herbin et al. Monitoring of atmospheric composition using the thermal infrared IASI/MetOp sounder. Atm. Chem. Phys., 9(16):6041-6054, 2009. doi: 10.5194/ acp-9-6041-2009.

[34] L.S. Rothman, I.E. Gordon, Y. Babikov, A. Barbe, D.C. Benner, P.F. Bernath et al. The HITRAN2012 molecular spectroscopic database. J. Quant. Spectrosc. E Radiat. Transfer, 130:4-50, 2013. doi: 10.1016/j.jqsrt.2013.07.002.

[35] N. Jacquinet-Husson, R. Armante, N.A. Scott, A. Chédin, L. Crépeau, C. Boutammine et al. The 2015 edition of the GEISA spectroscopic database. $J$. Mol. Spectrosc., 327:31 - 72, 2016. doi: 10.1016/j.jms.2016.06.007. New Visions of Spectroscopic Databases, Volume II.

[36] L.S. Rothman, I.E. Gordon, R.J. Barber, H. Dothe, R.R. Gamache, A. Goldman et al. HITEMP, the high-temperature molecular spectroscopic database. J. Quant. Spectrosc. \& Radiat. Transfer, 111(12-13):2139-2150, 2010 . doi: 10.1016/j.jqsrt.2010.05.001.

[37] J. Tennyson, S.N. Yurchenko, A.F. Al-Refaie, E.J. Barton, K.L. Chubb, P.A. Coles et al. The ExoMol database: Molecular line lists for exoplanet and other hot atmospheres. J. Mol. Spectrosc., 327:73 - 94, 2016. doi: 10.1016/j.jms.2016. 05.002. New Visions of Spectroscopic Databases, Volume II. 